IJMMS 29:10 (2002) 591-608

PII. S0161171202006361

http://ijmms.hindawi.com

(c) Hindawi Publishing Corp.

\title{
RELATIONSHIPS OF CONVOLUTION PRODUCTS, GENERALIZED TRANSFORMS, AND THE FIRST VARIATION ON FUNCTION SPACES
}

\author{
SEUNG JUN CHANG and JAE GIL CHOI
}

Received 9 December 2000

\begin{abstract}
We use a generalized Brownian motion process to define the generalized Fourier-Feynman transform, the convolution product, and the first variation. We then examine the various relationships that exist among the first variation, the generalized Fourier-Feynman transform, and the convolution product for functionals on function space that belong to a Banach algebra $S\left(L_{a b}[0, T]\right)$. These results subsume similar known results obtained by Park, Skoug, and Storvick (1998) for the standard Wiener process.
\end{abstract}

2000 Mathematics Subject Classification: 28C20.

1. Introduction. The concept of $L_{1}$ analytic Fourier-Feynman transform (FFT) was introduced by Brue in [1]. In [3], Cameron and Storvick introduced an $L_{2}$ analytic FFT. In [13], Johnson and Skoug developed an $L_{p}$ analytic FFT for $1 \leq p \leq 2$, which extended the results in $[1,3]$ and gave various relationships between the $L_{1}$ and $L_{2}$ theories. In [10], Huffman et al. defined a convolution product for functionals on Wiener space and obtained, in [11, 12], various results involving the FFT and the convolution product.

Both the FFT and the convolution product are defined in terms of a Feynman integral. In this paper, we extend the ideas of $[10,11,12]$ from the Wiener process to more general stochastic process. We note that the Wiener process is free of drift and is stationary in time. However, the stochastic process considered in this paper is subject to drift and nonstationary in time.

In Section 2, we consider the function space induced by a generalized Brownian motion process and define several concepts. In Section 3, we examine all relationships involving exactly two of three concepts of transform, convolution product and first variation of functionals in $S\left(L_{a b}[0, T]\right)$. In Section 4 , we examine all relationships involving all three of theses concepts where each concept is used exactly once.

2. Definitions and preliminaries. Let $D=[0, T]$ and let $(\Omega, \mathscr{B}, P)$ be a probability measure space. A real-valued stochastic process $Y$ on $(\Omega, \mathscr{B}, P)$ and $D$ is called a generalized Brownian motion process if $Y(0, \omega)=0$ a.e.; and for $0 \leq t_{0}<t_{1}<\cdots<t_{n} \leq T$, the $n$-dimensional random vector $\left(Y\left(t_{1}, \omega\right), \ldots, Y\left(t_{n}, \omega\right)\right)$ is normally distributed with the density function

$$
\begin{aligned}
K(\vec{t}, \vec{\eta})= & \left((2 \pi)^{n} \prod_{j=1}^{n}\left(b\left(t_{j}\right)-b\left(t_{j-1}\right)\right)\right)^{-1 / 2} \\
& \cdot \exp \left\{-\frac{1}{2} \sum_{j=1}^{n} \frac{\left(\left(\eta_{j}-a\left(t_{j}\right)\right)-\left(\eta_{j-1}-a\left(t_{j-1}\right)\right)\right)^{2}}{b\left(t_{j}\right)-b\left(t_{j-1}\right)}\right\},
\end{aligned}
$$


where $\vec{\eta}=\left(\eta_{1}, \ldots, \eta_{n}\right), \eta_{0}=0, \vec{t}=\left(t_{1}, \ldots, t_{n}\right), t_{0}=0$, and $a(t)$ is a continuous realvalued function of bounded variation with $a(0)=0$, and $b(t)$ is a strictly increasing, continuous real-valued function with $b(0)=0$.

As explained in [19, pages 18-20], $Y$ induces a probability measure $\mu$ on the measurable space $\left(\mathbb{R}^{D}, \mathscr{B}^{D}\right)$ where $\mathbb{R}^{D}$ is the space of all real-valued functions $x(t), t \in D$, and $\mathscr{B}^{D}$ is the smallest $\sigma$-algebra of subsets of $\mathbb{R}^{D}$ with respect to which all the coordinate evaluation maps, $e_{t}(x)=x(t)$, defined on $\mathbb{R}^{D}$ are measurable. The triple $\left(\mathbb{R}^{D}, \mathscr{B}^{D}, \mu\right)$ is a probability measure space.

We note that, the generalized Brownian motion process $Y$ determined by $a(\cdot)$ and $b(\cdot)$ is a Gaussian process with mean function $a(t)$ and covariance function $r(s, t)=$ $\min \{b(s), b(t)\}$. By [19, Theorem 14.2, page 187], the probability measure $\mu$ induced by $Y$, taking a separable version, is supported by $C_{a b}[0, T]$ (which is equivalent to the Banach space of continuous functions $x$ on $[0, T]$ with $x(0)=0$ under the sup norm). Hence $\left(C_{a b}[0, T], \mathscr{B}\left(C_{a b}[0, T]\right), \mu\right)$ is the function space induced by $Y$ where $\mathscr{B}\left(C_{a b}[0, T]\right)$ is the Borel $\sigma$-algebra of $C_{a b}[0, T]$. Note that we can also express $x$ in the form

$$
x(t)=w(b(t))+a(t), \quad 0 \leq t \leq T,
$$

where $w(\cdot)$ is the standard Brownian motion process [6, 7].

Let $L_{a b}[0, T]$ be the Hilbert space of functions on $[0, T]$ which are Lebesgue measurable and square integrable with respect to the Lebesgue Stieltjes measures on $[0, T]$ induced by $a(\cdot)$ and $b(\cdot)$, that is,

$$
L_{a b}[0, T]=\left\{v: \int_{0}^{T} v^{2}(s) d b(s)<\infty \text { and } \int_{0}^{T} v^{2}(s) d|a|(s)<\infty\right\},
$$

where $|a|$ denotes the total variation [6, 7].

A subset $B$ of $C_{a b}[0, T]$ is said to be scale-invariant measurable $[8,14]$ provided that $\rho B$ is $\mathscr{B}\left(C_{a b}[0, T]\right)$-measurable for all $\rho>0$, and a scale-invariant measurable set $N$ is said to be scale-invariant null set provided that $\mu(\rho N)=0$ for all $\rho>0$. A property that holds except on a scale-invariant null set, is said to hold on scale-invariant almost everywhere (s-a.e.). If two functionals $F$ and $G$ defined on $C_{a b}[0, T]$ are equal s-a.e., we write $F \approx G$.

We denote the function space integral of a $\mathscr{B}\left(C_{a b}[0, T]\right)$-measurable functional $F$ by

$$
E[F]=\int_{C_{a b}[0, T]} F(x) d \mu(x)
$$

whenever the integral exists.

We are now ready to state the definitions of the generalized analytic Feynman integral.

Definition 2.1. Let $\mathbb{C}$ denote the complex numbers. Let $\mathbb{C}_{+}=\{\lambda \in \mathbb{C}: \operatorname{Re} \lambda>0\}$ and $\tilde{\mathbb{C}}_{+}=\{\lambda \in \mathbb{C}: \lambda \neq 0$ and $\operatorname{Re} \lambda \geq 0\}$. Let $F: C_{a b}[0, T] \rightarrow \mathbb{C}$ be such that, for each $\lambda>0$, the function space integral

$$
J(\lambda)=\int_{C_{a b}[0, T]} F\left(\lambda^{-1 / 2} x\right) d \mu(x)
$$


exists for all $\lambda>0$. If there exists a function $J^{*}(\lambda)$ analytic in $\mathbb{C}_{+}$such that $J^{*}(\lambda)=J(\lambda)$ for all $\lambda>0$, then $J^{*}(\lambda)$ is defined to be the analytic function space integral of $F$ over $C_{a b}[0, T]$ with parameter $\lambda$, and for $\lambda \in \mathbb{C}_{+}$we write

$$
E^{a n_{\lambda}}[F]=J^{*}(\lambda)
$$

Let $q \neq 0$ be a real number and let $F$ be a functional such that $E^{a n_{\lambda}}[F]$ exists for all $\lambda \in \mathbb{C}_{+}$. If the following limit exists, we call it the generalized analytic Feynman integral of $F$ with parameter $q$ and we write

$$
E^{a n f_{q}}[F]=\lim _{\lambda \rightarrow-i q} E^{a n_{\lambda}}[F],
$$

where $\lambda$ approaches $-i q$ through $\mathbb{C}_{+}$.

Next we state the definition of the generalized FFT (GFFT).

DEFINITION 2.2. For $\lambda>0$ and $y \in C_{a b}[0, T]$, let

$$
T_{\lambda}(F)(y)=E_{x}^{a n_{\lambda}}[F(y+x)]
$$

In the standard Fourier theory, the integrals involved are often interpreted in the mean; a similar concept is useful in the FFT theory [13, page 104]. Let $p \in(1,2]$ and let $p$ and $p^{\prime}$ be related by $1 / p+1 / p^{\prime}=1$. Let $\left\{H_{n}\right\}$ and $H$ be scale-invariant measurable functionals such that for each $\rho>0$,

$$
\lim _{n \rightarrow \infty} E\left[\left|H_{n}(\rho y)-H(\rho y)\right|^{p^{\prime}}\right]=0 .
$$

Then we write

$$
H \approx \lim _{n \rightarrow \infty} . H_{n}
$$

and we call $H$ the scale-invariant limit in the mean of order $p^{\prime}$. A similar definition is understood when $n$ is replaced by the continuously varying parameter $\lambda$. Let real $q \neq 0$ be given. For $1<p \leq 2$, we define the $L_{p}$ analytic GFFT, $T_{q}^{(p)}(F)$ of $F$, by the formula $\left(\lambda \in \mathbb{C}_{+}\right)$

$$
T_{q}^{(p)}(F)(y)=\underset{\lambda \rightarrow-i q}{\lim . T_{\lambda}}(F)(y)
$$

if it exists. We define the $L_{1}$ analytic GFFT, $T_{q}^{(1)}(F)$ of $F$, by the formula $\left(\lambda \in \mathbb{C}_{+}\right)$

$$
T_{q}^{(1)}(F)(y)=\lim _{\lambda \rightarrow-i q} T_{\lambda}(F)(y)
$$

if it exists.

We note that for $1 \leq p \leq 2, T_{q}^{(p)}(F)$ is defined only s-a.e. We also note that if $T_{q}^{(p)}(F)$ exists and if $F \approx G$, then $T_{q}^{(p)}(G)$ exists and $T_{q}^{(p)}(G) \approx T_{q}^{(p)}(F)$. 
Definition 2.3. Let $F$ and $G$ be functionals on $C_{a b}[0, T]$. For $\lambda \in \tilde{\mathbb{C}}_{+}$we define the convolution product (if it exists) by

$$
(F * G)_{\lambda}(y)= \begin{cases}\int_{C_{a b}[0, T]}^{a n_{\lambda}} F\left(\frac{y+x}{\sqrt{2}}\right) G\left(\frac{y-x}{\sqrt{2}}\right) d \mu(x), \quad \lambda \in \mathbb{C}_{+} \\ \int_{C_{a b}[0, T]}^{a n f_{a}} F\left(\frac{y+x}{\sqrt{2}}\right) G\left(\frac{y-x}{\sqrt{2}}\right) d \mu(x), \quad \lambda=-i q .\end{cases}
$$

REMARK 2.4. When $\lambda=-i q$, we denote $(F * G)_{\lambda}$ by $(F * G)_{q}$.

We finish this section by giving the definition of the first variation $\delta F$ of the functional $F[2,5]$.

DEFINITION 2.5. Let $F$ be a $\mathscr{B}\left(C_{a b}[0, T]\right)$-measurable functional on $C_{a b}[0, T]$ and let $w \in C_{a b}[0, T]$. Then

$$
\delta F(x \mid w)=\left.\frac{\partial}{\partial h} F(x+h w)\right|_{h=0}
$$

(if it exists) is called the first variation of $F$.

The following analytic Feynman integration formula is used throughout:

$$
E^{a n f_{q}}[\exp \{i\langle v, x\rangle\}]=\exp \left\{-\frac{i}{2 q}\left(v^{2}, b\right)+i\left(\frac{i}{q}\right)^{1 / 2}(v, a)\right\}
$$

for all real $q \neq 0$, where $v \in L_{a b}[0, T],\langle v, x\rangle$ denotes the Paley-Wiener-Zygmund stochastic integral $\int_{0}^{T} v(s) d x(s)$, and $\left(v^{2}, b\right)$ denotes the Lebesgue Stieltjes integral $\int_{0}^{T} v^{2}(s) d b(s)$.

3. Relationships involving exactly two of three concepts of transform, convolution, and first variation. Let $M\left(L_{a b}[0, T]\right)$ be the space of $\mathbb{C}$-valued, countably additive finite Borel measures on $L_{a b}[0, T]$. The Banach algebra $S\left(L_{a b}[0, T]\right)$ consists of functionals $F$ on $C_{a b}[0, T]$ expressible in the form

$$
F(x)=\int_{L_{a b}[0, T]} \exp \{i\langle u, x\rangle\} d f(u),
$$

for s-a.e. $x \in C_{a b}[0, T]$, where $f$ is an element of $M\left(L_{a b}[0, T]\right)$. Further works on $S\left(L_{a b}[0, T]\right)$ show that it contains many functionals of interest in Feynman integration theory $[3,4,6,7,13,14,15,16,17]$.

Also, let

$A \equiv\left\{y \in C_{a b}[0, T]: y\right.$ is absolutely continuous on $[0, T]$ with $\left.y^{\prime} \in L_{a b}[0, T]\right\}$.

REMARK 3.1. Throughout, we choose the variance function $b(\cdot)$ which is strictly increasing such that the function $p$ defined by $p(t)=1 / b^{\prime}(t), p(0)=p(T)=0$ is of bounded variation on $[0, T]$. For any $u \in L_{a b}[0, T]$, let

$$
\|u\|_{b}=\left(\int_{0}^{T} u^{2}(s) d b(s)\right)^{1 / 2}=\sqrt{\left(u^{2}, b\right)},
$$


then $\|\cdot\|_{b}$ is a norm on $L_{a b}[0, T]$ since $\int_{0}^{T} u^{2}(s) d b(s)<\infty$. Hence we have for any $u, v \in L_{a b}[0, T]$,

$$
|(u v, b)| \leq\|u\|_{b}\|v\|_{b}
$$

This will insure that the first variation, $\delta F(\cdot \mid w)$ of $F$ in $S\left(L_{a b}[0, T]\right)$, that arises will exist for all $w \in A$ (see [9]).

Let $G$ in $S\left(L_{a b}[0, T]\right)$ be given by

$$
G(x)=\int_{L_{a b}[0, T]} \exp \{i\langle v, x\rangle\} d g(v)
$$

for s-a.e. $x \in C_{a b}[0, T]$ where $g \in M\left(L_{a b}[0, T]\right)$.

In our first lemma, we obtain a formula for the first variation of functionals in $S\left(L_{a b}[0, T]\right)$.

LEMMA 3.2. Let $F \in S\left(L_{a b}[0, T]\right)$ be given by (3.1) with $\int_{L_{a b}[0, T]}\|u\|_{b}|d f(u)|<\infty$. Then for each $w \in A$ and s-a.e. $y \in C_{a b}[0, T]$,

$$
\delta F(y \mid w)=\int_{L_{a b}[0, T]} i\langle u, w\rangle \exp \{i\langle u, y\rangle\} d f(u) .
$$

Furthermore, as a function of $y, \delta F(y \mid w)$ is an element of $S\left(L_{a b}[0, T]\right)$.

Proof. By using the definition of the first variation, we see that

$$
\begin{aligned}
\delta F(y \mid w) & =\left.\frac{\partial}{\partial h}\left(\int_{L_{a b}[0, T]} \exp \{i\langle u, y\rangle+i h\langle u, w\rangle\} d f(u)\right)\right|_{h=0} \\
& =\int_{L_{a b}[0, T]} i\langle u, w\rangle \exp \{\langle u, y\rangle\} d f(u)
\end{aligned}
$$

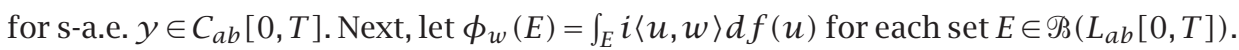
But

$$
\left\|\phi_{w}\right\| \leq \int_{L_{a b}[0, T]}|i\langle u, w\rangle||d f(u)| \leq M\left\|w^{\prime}\right\|_{b} \int_{L_{a b}[0, T]}\|u\|_{b}|d f(u)|<\infty,
$$

where $M=\sup _{t \in[0, T]} p(t)$. Hence $\delta F(y \mid w)=\int_{L_{a b}[0, T]} \exp \{i\langle u, y\rangle\} d \phi_{w}(u)$ is an element of $S\left(L_{a b}[0, T]\right)$.

In our next theorem, we obtain the transform of functional in $S\left(L_{a b}[0, T]\right)$.

THEOREM 3.3. Let $F \in S\left(L_{a b}[0, T]\right)$ be given by (3.1) and let $p \in[1,2]$ be given. Then the analytic generalized Fourier-Feynman transform $T_{q}^{(p)}(F)$ exists for all real $q \neq 0$ and is given by the formula

$$
T_{q}^{(p)}(F)(y)=\int_{L_{a b}[0, T]} \exp \left\{i\langle u, y\rangle-\frac{i}{2 q}\left(u^{2}, b\right)+i\left(\frac{i}{q}\right)^{1 / 2}(u, a)\right\} d f(u)
$$

for s-a.e. $y \in C_{a b}[0, T]$. 
Proof. By (2.8), the Fubini theorem, and (2.15), we have, for all $\lambda>0$,

$$
\begin{aligned}
T_{\lambda}(F)(y) & =\int_{C_{a b}[0, T]}^{a n_{\lambda}} F(y+x) d \mu(x) \\
& =\int_{L_{a b}[0, T]} \int_{C_{a b}[0, T]}^{a n_{\lambda}} \exp \{i\langle u, y\rangle+i\langle u, x\rangle\} d \mu(x) d f(u) \\
& =\int_{L_{a b}[0, T]} \exp \left\{i\langle u, y\rangle-\frac{1}{2 \lambda}\left(u^{2}, b\right)+\frac{i}{\sqrt{\lambda}}(u, a)\right\} d f(u)
\end{aligned}
$$

for s-a.e. $y \in C_{a b}[0, T]$. But the last equation above is analytic throughout $\mathbb{C}_{+}$and is continuous on $\tilde{\mathbb{C}}_{+}$, since $f$ is a finite Borel measure. Thus (3.9) is established.

In the following theorem, we obtain the convolution product of functionals in $S\left(L_{a b}[0, T]\right)$.

THEOREM 3.4. Let $F \in S\left(L_{a b}[0, T]\right)$ be given by (3.1), and let $G \in S\left(L_{a b}[0, T]\right)$ be given by (3.5). Then their convolution product $(F * G)_{q}$ exists for all real $q \neq 0$ and is given by the formula

$$
\begin{aligned}
(F * G)_{q}(y)=\int_{L_{a b}^{2}[0, T]} \exp \{ & \frac{i}{\sqrt{2}}\langle u+v, y\rangle-\frac{i}{4 q}\left((u-v)^{2}, b\right) \\
& \left.+i\left(\frac{i}{2 q}\right)^{1 / 2}(u-v, a)\right\} d f(u) d g(v)
\end{aligned}
$$

for s-a.e. $y \in C_{a b}[0, T]$.

Proof. By using (2.13), the Fubini theorem, and (2.15), we have that for all $\lambda>0$,

$$
\begin{aligned}
(F * & G)_{\lambda}(y) \\
& =\int_{C_{a b}[0, T]}^{a n_{\lambda}} F\left(\frac{y+x}{\sqrt{2}}\right) G\left(\frac{y-x}{\sqrt{2}}\right) d \mu(x) \\
& =\int_{L_{a b}^{2}[0, T]} \int_{C_{a b}[0, T]}^{a n_{\lambda}} \exp \left\{\frac{i}{\sqrt{2}}\langle u-v, x\rangle+\frac{i}{\sqrt{2}}\langle u+v, y\rangle\right\} d \mu(x) d f(u) d g(v) \\
& =\int_{L_{a b}^{2}[0, T]} \exp \left\{\frac{i}{\sqrt{2}}\langle u+v, y\rangle-\frac{1}{4 \lambda}\left((u-v)^{2}, b\right)+\frac{i}{\sqrt{2 \lambda}}(u-v, a)\right\} d f(u) d g(v)
\end{aligned}
$$

for s-a.e. $y \in C_{a b}[0, T]$. But the last equation above is analytic throughout $\mathbb{C}_{+}$, and is continuous on $\tilde{\mathbb{C}}_{+}$. Thus we have the desired result.

Next, we obtain the transform of the convolution product.

THEOREM 3.5. Let $F$ and $G$ be given as in Theorem 3.4 and $p \in[1,2]$. Then for all real $q \neq 0, T_{q}^{(p)}\left((F * G)_{q}\right)$ exists and is given by the formula

$$
T_{q}^{(p)}\left((F * G)_{q}\right)(y)=T_{q}^{(p)}\left(F_{1}\right)\left(\frac{y}{\sqrt{2}}\right) T_{q}^{(p)}\left(G_{1}\right)\left(\frac{y}{\sqrt{2}}\right)
$$

for s-a.e. $y \in C_{a b}[0, T]$, where $F_{1}$ and $G_{1}$ are given by (3.15) below. 
Proof. Let $p \in[1,2]$ and $q \in \mathbb{R}-\{0\}$. Using (2.15), (3.9), and (3.11), we see that

$$
\begin{aligned}
T_{q}^{(p)}( & \left.(F * G)_{q}\right)(y) \\
= & \int_{C_{a b}[0, T]}^{a n f_{q}}(F * G)_{q}(y+z) d \mu(z) \\
= & \int_{C_{a b}^{2}[0, T]}^{a n f_{q}} F\left(\frac{(y+z)+x}{\sqrt{2}}\right) G\left(\frac{(y+z)-x}{\sqrt{2}}\right) d \mu(x) d \mu(z) \\
= & \int_{L_{a b}^{2}[0, T]} \exp \left\{-\frac{i}{4 q}\left((u-v)^{2}, b\right)+i\left(\frac{i}{2 q}\right)^{1 / 2}(u-v, a)\right\} \\
& \cdot \int_{C_{a b}[0, T]}^{a n f_{q}} \exp \left\{\frac{i}{\sqrt{2}}\langle u+v, z\rangle+\frac{i}{\sqrt{2}}\langle u+v, y\rangle\right\} d \mu(z) d f(u) d g(v) \\
= & \int_{L_{a b}^{2}[0, T]} \exp \left\{-\frac{i}{4 q}\left((u-v)^{2}, b\right)+i\left(\frac{i}{2 q}\right)^{1 / 2}(u-v, a)-\frac{i}{4 q}\left((u+v)^{2}, b\right)\right. \\
= & \int_{L_{a b}[0, T]} \exp \left\{\frac{i}{\sqrt{2}}\langle u, y\rangle-\frac{i}{2 q}\left(u^{2}, b\right)+i\left(\frac{i}{q}\right)^{1 / 2}(u, a)\right\} d f_{1}(u) \\
& \cdot \int_{L_{a b}[0, T]} \exp \left\{\frac{i}{\sqrt{2}}\langle v, y\rangle-\frac{i}{2 q}\left(v^{2}, b\right)+i\left(\frac{i}{q}\right)^{1 / 2}(v, a)\right\} d g_{1}(v) \\
= & T_{q}^{(p)}\left(F_{1}\right)\left(\frac{y}{\sqrt{2}}\right) T_{q}^{(p)}\left(G_{1}\right)\left(\frac{y}{\sqrt{2}}\right)
\end{aligned}
$$

for s-a.e. $y \in C_{a b}[0, T]$, where

$$
\begin{aligned}
& F_{1}(y)=\int_{L_{a b}[0, T]} \exp \{i\langle u, y\rangle\} d f_{1}(u), \\
& G_{1}(y)=\int_{L_{a b}[0, T]} \exp \{i\langle v, y\rangle\} d g_{1}(v), \\
& f_{1}(E)=\int_{E} \exp \left\{-i\left(\frac{i}{q}\right)^{1 / 2}(u, a)+2 i\left(\frac{i}{2 q}\right)^{1 / 2}(u, a)\right\} d f(u), \\
& g_{1}(E)=\int_{E} \exp \left\{-i\left(\frac{i}{q}\right)^{1 / 2}(v, a)\right\} d g(v),
\end{aligned}
$$

for every $E \in \mathscr{B}\left(L_{a b}[0, T]\right)$, and so $\left\|f_{1}\right\| \leq\|f\|$ and $\left\|g_{1}\right\| \leq\|g\|$.

In our next theorem, we have the convolution product of transforms of $F$ and $G$ in $S\left(L_{a b}[0, T]\right)$.

THEOREM 3.6. Let $F, G$, and $p$ be given as in Theorem 3.5. Then for all real $q \neq 0$, $\left(T_{q}^{(p)}(F) * T_{q}^{(p)}(G)\right)_{-q}(y)$ exists and is given by the formula

$$
\left(T_{q}^{(p)}(F) * T_{q}^{(p)}(G)\right)_{-q}(y)=T_{q}^{(p)}\left(F_{2}\left(\frac{\cdot}{\sqrt{2}}\right) G_{2}\left(\frac{\cdot}{\sqrt{2}}\right)\right)(y)
$$

for s-a.e. $y \in C_{a b}[0, T]$ where $F_{2}$ and $G_{2}$ are given by (3.19) below. 
Proof. By using (3.9), (3.11), and the Fubini theorem, we have, for s-a.e. $y \in$ $C_{a b}[0, T]$,

$$
\begin{aligned}
\left(T_{q}^{(p)}(F) * T_{q}^{(p)}(G)\right)_{-q}(y) & \int_{C_{a b}[0, T]}^{a n f_{-q}} T_{q}^{(p)}(F)\left(\frac{y+x}{\sqrt{2}}\right) T_{q}^{(p)}(G)\left(\frac{y-x}{\sqrt{2}}\right) d \mu(x) \\
= & \int_{L_{a b}^{2}[0, T]} \exp \left\{\frac{i}{\sqrt{2}}\langle u+v, y\rangle-\frac{i}{2 q}\left(u^{2}+v^{2}, b\right)+i\left(\frac{i}{q}\right)^{1 / 2}(u+v, a)\right\} \\
& \cdot\left[\int_{C_{a b}[0, T]}^{a n f_{-q}} \exp \left\{\frac{i}{\sqrt{2}}\langle u-v, x\rangle\right\} d \mu(x)\right] d f(u) d g(v) \\
= & \int_{L_{a b}^{2}[0, T]} \exp \left\{\frac{i}{\sqrt{2}}\langle u+v, y\rangle-\frac{i}{2 q}\left(u^{2}+v^{2}, b\right)+i\left(\frac{i}{q}\right)^{1 / 2}(u+v, a)\right. \\
= & \int_{L_{a b}^{2}[0, T]} \exp \left\{\frac { i } { \sqrt { 2 } } \left\langle(u+v, y\rangle-\frac{i}{4 q}\left((u+v)^{2}, b\right)+i\left(\frac{i}{2 q}\right)^{1 / 2}(u+v, a)\right.\right. \\
& -i\left(\frac{i}{2 q}\right)^{1 / 2}(u+v, a)+i\left(-\frac{i}{2 q}\right)^{1 / 2}(u-v, a) \\
& \left.+i\left(\frac{i}{q}\right)^{1 / 2}(u+v, a)\right\} d f(u) d g(v) \\
= & \int_{L_{a b}^{2}[0, T]} \exp \left\{\frac{i}{\sqrt{2}}\langle u+v, y\rangle-\frac{i}{4 q}\left((u+v)^{2}, b\right)\right. \\
& \left.+i\left(\frac{i}{2 q}\right)^{1 / 2}(u+v, a)\right\} d f_{2}(u) d g_{2}(v) \\
& \cdot\left(F_{2}\left(\frac{i}{\sqrt{2}}\right) G_{2}\left(\frac{\cdot}{\sqrt{2}}\right)\right)(y),
\end{aligned}
$$

where

$$
\begin{aligned}
& F_{2}(y)=\int_{L_{a b}[0, T]} \exp \{i\langle u, y\rangle\} d f_{2}(u), \\
& G_{2}(y)=\int_{L_{a b}[0, T]} \exp \{i\langle v, y\rangle\} d g_{2}(v), \\
& f_{2}(E)=\int_{E} \exp \left\{i\left(-\left(\frac{i}{2 q}\right)^{1 / 2}+\left(-\frac{i}{2 q}\right)^{1 / 2}+\left(\frac{i}{q}\right)^{1 / 2}\right)(u, a)\right\} d f(u), \\
& g_{2}(E)=\int_{E} \exp \left\{i\left(-\left(\frac{i}{2 q}\right)^{1 / 2}-\left(-\frac{i}{2 q}\right)^{1 / 2}+\left(\frac{i}{q}\right)^{1 / 2}\right)(v, a)\right\} d g(v),
\end{aligned}
$$

for every $E \in \mathscr{B}\left(L_{a b}[0, T]\right)$, and so $\left\|f_{2}\right\| \leq\|f\|$ and $\left\|g_{2}\right\| \leq\|g\|$.

In the next theorem, we obtain that the transform with respect to the first argument of the variation equals the variation of the transform. 
THEOREM 3.7. Let $F$ be given as in Lemma 3.2, $p \in[1,2], q \in \mathbb{R}-\{0\}$, and $w \in A$ be given. Then

$$
T_{q}^{(p)}(\delta F(\cdot \mid w))(y)=\delta T_{q}^{(p)}(F)(y \mid w)
$$

for s-a.e. $y \in C_{a b}[0, T]$.

Also, both expressions in (3.21) are given by the expression

$$
\int_{L_{a b}[0, T]} i\langle u, w\rangle \exp \left\{i\langle u, y\rangle-\frac{i}{2 q}\left(u^{2}, b\right)+i\left(\frac{i}{q}\right)^{1 / 2}(u, a)\right\} d f(u) .
$$

Proof. By using (3.6), the Fubini theorem, (2.15), and (3.9), we have that

$$
\begin{aligned}
T_{q}^{(p)}( & \delta F(\cdot \mid w))(y) \\
& =\int_{C_{a b}[0, T]}^{a n f_{q}} \delta F(y+x \mid w) d \mu(x) \\
& =\int_{C_{a b}[0, T]}^{a n f_{q}} \int_{L_{a b}[0, T]} i\langle u, w\rangle \exp \{i\langle u, y+x\rangle\} d f(u) d \mu(x) \\
& =\int_{L_{a b}[0, T]} i\langle u, w\rangle \exp \{i\langle u, y\rangle\}\left[\int_{C_{a b}[0, T]}^{a n f_{q}} \exp \{i\langle u, x\rangle\} d \mu(x)\right] d f(u) \\
& =\int_{L_{a b}[0, T]} i\langle u, w\rangle \exp \left\{i\langle u, y\rangle-\frac{i}{2 q}\left(u^{2}, b\right)+i\left(\frac{i}{q}\right)^{1 / 2}(u, a)\right\} d f(u) \\
& =\left.\int_{L_{a b}[0, T]} \frac{\partial}{\partial h}\left(\exp \left\{i\langle u, y+h w\rangle-\frac{i}{2 q}\left(u^{2}, b\right)+i\left(\frac{i}{q}\right)^{1 / 2}(u, a)\right\}\right)\right|_{h=0} d f(u) \\
& =\left.\frac{\partial}{\partial h}\left(T_{q}^{(p)}(F)(y+h w)\right)\right|_{h=0} \\
& =\delta T_{q}^{(p)}(F)(y \mid w)
\end{aligned}
$$

for s-a.e. $y \in C_{a b}[0, T]$ as desired.

In the next theorem, we obtain the transform with respect to the second argument of the variation.

THEOREM 3.8. Let $F, p, q$, and $w$ be given as in Theorem 3.7. Then, for s-a.e. $y \in$ $C_{a b}[0, T]$,

$$
T_{q}^{(p)}(\delta F(y \mid \cdot))(w)=\delta F(y \mid w)+i\left(\frac{i}{q}\right)^{1 / 2} \int_{L_{a b}[0, T]}(u, a) \exp \{i\langle u, y\rangle\} d f(u) .
$$

Proof. Using (2.11) and (3.6), we obtain

$$
\begin{aligned}
T_{q}^{(p)}(\delta F(y \mid \cdot))(w) & =\int_{C_{a b}[0, T]}^{a n f_{q}} \delta F(y \mid w+x) d \mu(x) \\
& =\int_{C_{a b}[0, T]}^{a n f_{q}} \int_{L_{a b}[0, T]} i\langle u, w+x\rangle \exp \{i\langle u, y\rangle\} d f(u) d \mu(x)
\end{aligned}
$$




$$
\begin{aligned}
& =i \int_{L_{a b}[0, T]} \exp \{i\langle u, y\rangle\}\left[\int_{C_{a b}[0, T]}^{a n f_{q}}\langle u, w+x\rangle d \mu(x)\right] d f(u) \\
& =i \int_{L_{a b}[0, T]}\left(\langle u, w\rangle+\left(\frac{i}{q}\right)^{1 / 2}(u, a)\right) \exp \{i\langle u, y\rangle\} d f(u) \\
& =\delta F(y \mid w)+i\left(\frac{i}{q}\right)^{1 / 2} \int_{L_{a b}[0, T]}(u, a) \exp \{i\langle u, y\rangle\} d f(u)
\end{aligned}
$$

for s-a.e. $y \in C_{a b}[0, T]$. In particular, if $a \in A$ then $(u, a)=\langle u, a\rangle$ and so

$$
T_{q}^{(p)}(\delta F(y \mid \cdot))(w)=\delta F(y \mid w)+\left(\frac{i}{q}\right)^{1 / 2} \delta F(y \mid a) .
$$

In our next theorem, we obtain the first variation of convolution product of functionals $F$ and $G$ in $S\left(L_{a b}[0, T]\right)$.

THEOREM 3.9. Let $F, p, q$, and $w$ be given as in Theorem 3.7 and let $G$ be given by (3.5) with $\int_{L_{a b}[0, T]}\|v\|_{b}|d g(v)|<\infty$. Then for s-a.e. $y \in C_{a b}[0, T]$, we obtain the formula

$$
\begin{aligned}
\delta(F * G)_{q}(y \mid w) & \\
=\int_{L_{a b}^{2}[0, T]} \frac{i}{\sqrt{2}}\langle u+v, w\rangle \exp \{ & \frac{i}{\sqrt{2}}\langle u+v, y\rangle-\frac{i}{4 q}\left((u-v)^{2}, b\right) \\
& \left.+i\left(\frac{i}{2 q}\right)^{1 / 2}(u-v, a)\right\} d f(u) d g(v)
\end{aligned}
$$

for s-a.e. $y \in C_{a b}[0, T]$.

PROoF. The proof of (3.27) can be obtained by using (3.6) and (3.11).

In our next theorem, we obtain the convolution product of the first variation with respect to the first argument.

THEOREM 3.10. Let $F, G, p, q$, and $w$ be given as in Theorem 3.9. Then for s-a.e. $y \in C_{a b}[0, T],(\delta F(\cdot \mid w) * \delta G(\cdot \mid w))_{q}(y)$ exists and is given by the formula

$$
\begin{aligned}
(\delta F(\cdot \mid w) * \delta G(\cdot \mid w))_{q}(y) & \\
=-\int_{L_{a b}^{2}[0, T]}\langle u, w\rangle\langle v, w\rangle \exp \{ & \frac{i}{\sqrt{2}}\langle u+v, y\rangle-\frac{i}{4 q}\left((u-v)^{2}, b\right) \\
& \left.+i\left(\frac{i}{2 q}\right)^{1 / 2}(u-v, a)\right\} d f(u) d g(v) .
\end{aligned}
$$

Proof. By using (2.13), (3.6), the Fubini theorem, and (2.15), we have

$$
\begin{aligned}
& (\delta F(\cdot \mid w) * \delta G(\cdot \mid w))_{q}(y) \\
& \quad=\int_{C_{a b}[0, T]}^{a n f_{q}} \delta F\left(\frac{y+x}{\sqrt{2}} \mid w\right) \delta G\left(\frac{y-x}{\sqrt{2}} \mid w\right) d \mu(x)
\end{aligned}
$$




$$
\begin{gathered}
=\int_{C_{a b}[0, T]}^{a n f_{q}} \int_{L_{a b}[0, T]} i\langle u, w\rangle \exp \left\{i\left\langle u, \frac{y+x}{\sqrt{2}}\right\rangle\right\} d f(u) \\
\cdot \int_{L_{a b}[0, T]} i\langle v, w\rangle \exp \left\{i\left\langle v, \frac{y-x}{\sqrt{2}}\right\rangle\right\} d g(v) d \mu(x) \\
=-\int_{L_{a b}^{2}[0, T]} \int_{C_{a b}[0, T]}^{a n f_{q}}\langle u, w\rangle\langle v, w\rangle \\
\cdot \exp \left\{\frac{i}{\sqrt{2}}\langle u-v, x\rangle+\frac{i}{\sqrt{2}}\langle u+v, y\rangle\right\} d \mu(x) d f(u) d g(v) \\
=-\int_{L_{a b}^{2}[0, T]}\langle u, w\rangle\langle v, w\rangle \exp \left\{\frac{i}{\sqrt{2}}\langle u+v, y\rangle-\frac{i}{4 q}\left((u-v)^{2}, b\right)\right. \\
\left.+i\left(\frac{i}{2 q}\right)^{1 / 2}(u-v, a)\right\} d f(u) d g(v)
\end{gathered}
$$

for s-a.e. $y \in C_{a b}[0, T]$.

In our next theorem, we obtain the convolution product of the first variation with respect to the second argument.

THEOREM 3.11. Let $F, G, p, q$, and $w$ be given as in Theorem 3.9. Then

$$
\begin{aligned}
(\delta F(y \mid \cdot) * \delta G(y \mid \cdot))_{q}(w) \\
=\frac{i}{2 q} \int_{L_{a b}^{2}[0, T]}(u v, b) \exp \{i\langle u+v, y\rangle\} d f(u) d g(v) \\
\quad+\left(\delta F\left(y \mid \frac{w}{\sqrt{2}}\right)+i\left(\frac{i}{q}\right)^{1 / 2} \int_{L_{a b}[0, T]}\left(u, \frac{a}{\sqrt{2}}\right) \exp \{i\langle u, y\rangle\} d f(u)\right) \\
\quad \cdot\left(\delta G\left(y \mid \frac{w}{\sqrt{2}}\right)-i\left(\frac{i}{q}\right)^{1 / 2} \int_{L_{a b}[0, T]}\left(v, \frac{a}{\sqrt{2}}\right) \exp \{i\langle v, y\rangle\} d g(v)\right)
\end{aligned}
$$

for s-a.e. $y \in C_{a b}[0, T]$.

Proof. For each $u, v \in L_{a b}[0, T]$, we have

$$
\int_{C_{a b}[0, T]}^{a n f_{q}}\langle u, x\rangle\langle v, x\rangle d \mu(x)=\frac{i}{q}(u, a)(v, a)+\frac{i}{q}(u v, b) .
$$

But, by using (2.13), (3.6), the Fubini theorem, and (3.31), we have

$$
\begin{aligned}
& (\delta F(y \mid \cdot) * \delta G(y \mid \cdot))_{q}(w) \\
& \quad=\int_{C_{a b}[0, T]}^{a n f_{q}} \delta F\left(y \mid \frac{w+x}{\sqrt{2}}\right) \delta G\left(y \mid \frac{w-x}{\sqrt{2}}\right) d \mu(x) \\
& \quad=-\frac{1}{2} \int_{L_{a b}^{2}[0, T]} \int_{C_{a b}[0, T]}^{a n f_{q}}\langle u, w+x\rangle\langle v, w-x\rangle \exp \{i\langle u+v, y\rangle\} d \mu(x) d f(u) d g(v)
\end{aligned}
$$




$$
\begin{aligned}
= & -\frac{1}{2} \int_{L_{a b}^{2}[0, T]}\left[\left(\left(\frac{i}{q}\right)^{1 / 2}(u, a)+\langle u, w\rangle\right)\left(\langle v, w\rangle-\left(\frac{i}{q}\right)^{1 / 2}(v, a)\right)-\frac{i}{q}(u v, b)\right] \\
& \cdot \exp \{i\langle u+v, y\rangle\} d f(u) d g(v) \\
= & \int_{L_{a b}[0, T]} \frac{i}{\sqrt{2}}\left(\langle u, w\rangle+\left(\frac{i}{q}\right)^{1 / 2}(u, a)\right) \exp \{i\langle u, y\rangle\} d f(u) \\
& \cdot \int_{L_{a b}[0, T]} \frac{i}{\sqrt{2}}\left(\langle v, w\rangle-\left(\frac{i}{q}\right)^{1 / 2}(v, a)\right) \exp \{i\langle v, y\rangle\} d g(v) \\
& +\frac{i}{2 q} \int_{L_{a b}^{2}[0, T]}(u v, b) \exp \{i\langle u+v, y\rangle\} d f(u) d g(v) \\
= & \left(\delta F\left(y \mid \frac{w}{\sqrt{2}}\right)+i\left(\frac{i}{q}\right)^{1 / 2} \int_{L_{a b}[0, T]}\left(u, \frac{a}{\sqrt{2}}\right) \exp \{\langle u, y\rangle\} d f(u)\right) \\
& \cdot\left(\delta G\left(y \mid \frac{w}{\sqrt{2}}\right)-i\left(\frac{i}{q}\right)^{1 / 2} \int_{L_{a b}[0, T]}\left(v, \frac{a}{\sqrt{2}}\right) \exp \{\langle v, y\rangle\} d g(v)\right) \\
& +\frac{i}{2 q} \int_{L_{a b}^{2}[0, T]}(u v, b) \exp \{i\langle u+v, y\rangle\} d f(u) d g(v)
\end{aligned}
$$

for s-a.e. $y \in C_{a b}[0, T]$. In particular, if $a \in A$ then $(u, a / \sqrt{2})=\langle u, a / \sqrt{2}\rangle$ and $(v$, $a / \sqrt{2})=\langle v, a / \sqrt{2}\rangle$ and so we have

$$
\begin{aligned}
(\delta F(y \mid \cdot) * \delta G(y \mid \cdot))_{q}(w) \\
=\left(\delta F\left(y \mid \frac{w}{\sqrt{2}}\right)+i\left(\frac{i}{q}\right)^{1 / 2} \delta F\left(y, \frac{a}{\sqrt{2}}\right)\right)\left(\delta G\left(y \mid \frac{w}{\sqrt{2}}\right)-i\left(\frac{i}{q}\right)^{1 / 2} \delta G\left(y, \frac{a}{\sqrt{2}}\right)\right) \\
\quad+\frac{i}{2 q} \int_{L_{a b}^{2}[0, T]}(u v, b) \exp \{i\langle u+v, y\rangle\} d f(u) d g(v) .
\end{aligned}
$$

Also, by using (3.24), the alternative expression in (3.30) is given by

$$
\begin{aligned}
-T_{q}^{(p)}\left(\delta F\left(y \mid \frac{\cdot}{\sqrt{2}}\right)\right)(w) T_{q}^{(p)}\left(\delta G\left(y \mid \frac{\cdot}{\sqrt{2}}\right)\right)(-w) \\
+\frac{i}{2 q} \int_{L_{a b}^{2}[0, T]}(u v, b) \exp \{i\langle u+v, y\rangle\} d f(u) d g(v) .
\end{aligned}
$$

Thus we have the desired result.

4. Relationships involving three concepts. In this section, we look at all the relationships involving the transform, the convolution, and the first variation where each operation is used exactly once.

In our next theorem, we obtain the formula for transform with respect to the first argument of the variation of the convolution product which equals the variation of the transform of the convolution product. 
THEOREM 4.1. Let $F, G, p, q$, and $w$ be given as in Theorem 3.9. Then for s-a.e. $y \in C_{a b}[0, T], T_{q}^{(p)}\left(\delta(F * G)_{q}(\cdot \mid w)\right)(y)$ exists and is given by the formula

$$
\begin{aligned}
T_{q}^{(p)}\left(\delta(F * G)_{q}(\cdot \mid w)\right)(y)= & T_{q}^{(p)}\left(F_{1}\right)\left(\frac{y}{\sqrt{2}}\right) T_{q}^{(p)}\left(\delta G_{1}\left(\cdot \mid \frac{w}{\sqrt{2}}\right)\right)\left(\frac{y}{\sqrt{2}}\right) \\
& +T_{q}^{(p)}\left(\delta F_{1}\left(\cdot \mid \frac{w}{\sqrt{2}}\right)\right)\left(\frac{y}{\sqrt{2}}\right) T_{q}^{(p)}\left(G_{1}\right)\left(\frac{y}{\sqrt{2}}\right)
\end{aligned}
$$

where $F_{1}$ and $G_{1}$ are given by (3.15).

Proof. By using (3.21) we have

$$
T_{q}^{(p)}\left(\delta(F * G)_{q}(\cdot \mid w)\right)(y)=\delta T_{q}^{(p)}\left((F * G)_{q}\right)(y \mid w) .
$$

Also, using (3.6) and (3.13), we obtain

$$
\begin{aligned}
\delta T_{q}^{(p)}( & \left.(F * G)_{q}\right)(y \mid w) \\
= & \left.\frac{\partial}{\partial h}\left(T_{q}^{(p)}\left(F_{1}\right)\left(\frac{y+h w}{\sqrt{2}}\right) T_{q}^{(p)}\left(G_{1}\right)\left(\frac{y+h w}{\sqrt{2}}\right)\right)\right|_{h=0} \\
= & \frac{\partial}{\partial h}\left[\int_{L_{a b}[0, T]} \exp \left\{\frac{i}{\sqrt{2}}\langle u, y+h w\rangle-\frac{i}{2 q}\left(u^{2}, b\right)+i\left(\frac{i}{q}\right)^{1 / 2}(u, a)\right\} d f_{1}(u)\right. \\
& \left.\cdot \int_{L_{a b}[0, T]} \exp \left\{\frac{i}{\sqrt{2}}\langle v, y+h w\rangle-\frac{i}{2 q}\left(v^{2}, b\right)+i\left(\frac{i}{q}\right)^{1 / 2}(v, a)\right\} d g_{1}(v)\right]\left.\right|_{h=0} \\
= & \int_{L_{a b}[0, T]} \exp \left\{\frac{i}{\sqrt{2}}\langle u, y\rangle-\frac{i}{2 q}\left(u^{2}, b\right)+i\left(\frac{i}{q}\right)^{1 / 2}(u, a)\right\} d f_{1}(u) \\
& \cdot \int_{L_{a b}[0, T]} \frac{i}{\sqrt{2}}\langle v, w\rangle \exp \left\{\frac{i}{\sqrt{2}}\langle v, y\rangle-\frac{i}{2 q}\left(v^{2}, b\right)+i\left(\frac{i}{q}\right)^{1 / 2}(v, a)\right\} d g_{1}(v) \\
& +\int_{L_{a b}[0, T]} \frac{i}{\sqrt{2}}\langle u, w\rangle \exp \left\{\frac{i}{\sqrt{2}}\langle u, y\rangle-\frac{i}{2 q}\left(u^{2}, b\right)+i\left(\frac{i}{q}\right)^{1 / 2}(u, a)\right\} d f_{1}(u) \\
& \cdot \int_{L_{a b}[0, T]} \exp \left\{\frac{i}{\sqrt{2}}\langle v, y\rangle-\frac{i}{2 q}\left(v^{2}, b\right)+i\left(\frac{i}{q}\right)^{1 / 2}(v, a)\right\} d g_{1}(v) \\
= & T_{q}^{(p)}\left(F_{1}\right)\left(\frac{y}{\sqrt{2}}\right) \delta T_{q}^{(p)}\left(G_{1}\right)\left(\frac{y}{\sqrt{2}} \mid \frac{w}{\sqrt{2}}\right)+\delta T_{q}^{(p)}\left(F_{1}\right)\left(\frac{y}{\sqrt{2}} \mid \frac{w}{\sqrt{2}}\right) T_{q}^{(p)}\left(G_{1}\right)\left(\frac{y}{\sqrt{2}}\right) \\
= & T_{q}^{(p)}\left(F_{1}\right)\left(\frac{y}{\sqrt{2}}\right) T_{q}^{(p)}\left(\delta G_{1}\left(\cdot \mid \frac{w}{\sqrt{2}}\right)\right)\left(\frac{y}{\sqrt{2}}\right) \\
& +T_{q}^{(p)}\left(\delta F_{1}\left(\cdot \mid \frac{w}{\sqrt{2}}\right)\right)\left(\frac{y}{\sqrt{2}}\right) T_{q}^{(p)}\left(G_{1}\right)\left(\frac{y}{\sqrt{2}}\right)
\end{aligned}
$$

for s-a.e. $y \in C_{a b}[0, T]$.

In our next theorem, we obtain the transform with respect to the second argument of the variation of the convolution product. 
THEOREM 4.2. Let $F, G, p, q$, and $w$ be given as in Theorem 4.1. Then for s-a.e. $y \in C_{a b}[0, T]$,

$$
\begin{aligned}
& T_{q}^{(p)}\left(\delta(F * G)_{q}(y \mid \cdot)\right)(w) \\
&= \frac{i}{\sqrt{2}} \int_{L_{a b}^{2}[0, T]}\left[\langle u+v, w\rangle+\left(\frac{i}{q}\right)^{1 / 2}(u+v, a)\right] \\
& \quad \cdot \exp \left\{\frac{i}{\sqrt{2}}\langle u+v, y\rangle-\frac{i}{4 q}\left((u-v)^{2}, b\right)+i\left(\frac{i}{2 q}\right)^{1 / 2}(u-v, a)\right\} d f(u) d g(v) \\
&=\delta(F * G)_{q}(y \mid w)+\frac{i}{\sqrt{2}}\left(\frac{i}{q}\right)^{1 / 2} \\
& \quad \cdot \int_{L_{a b}^{2}[0, T]}(u+v, a) \exp \left\{\frac{i}{\sqrt{2}}\langle u+v, y\rangle-\frac{i}{4 q}\left((u-v)^{2}, b\right)\right. \\
&\left.\quad+i\left(\frac{i}{2 q}\right)^{1 / 2}(u-v, a)\right\} d f(u) d g(v) .
\end{aligned}
$$

Proof. By using (3.11), (3.24), and (3.27), we obtain (4.4) above. In particular, if $a \in A$, then $(u+v, a)=\langle u+v, a\rangle$ and hence we have

$$
T_{q}^{(p)}\left(\delta(F * G)_{q}(y \mid \cdot)\right)(w)=\delta(F * G)_{q}(y \mid w)+\left(\frac{i}{q}\right)^{1 / 2} \delta(F * G)_{q}(y \mid a) .
$$

Now we obtain formulas for the transforms of the convolution product with respect to the first argument of the variations.

THEOREM 4.3. Let $F, G, p, q$, and $w$ be given as in Theorem 4.1. Then, for s-a.e. $y \in C_{a b}[0, T]$,

$$
\begin{aligned}
T_{q}^{(p)} & \left((\delta F(\cdot \mid w) * \delta G(\cdot \mid w))_{q}\right)(y) \\
& =T_{q}^{(p)}\left(\delta F_{1}(\cdot \mid w)\right)\left(\frac{y}{\sqrt{2}}\right) T_{q}^{(p)}\left(\delta G_{1}(\cdot \mid w)\right)\left(\frac{y}{\sqrt{2}}\right) \\
& =\delta T_{q}^{(p)}\left(F_{1}\right)\left(\frac{y}{\sqrt{2}} \mid w\right) \delta T_{q}^{(p)}\left(G_{1}\right)\left(\frac{y}{\sqrt{2}} \mid w\right),
\end{aligned}
$$

where $F_{1}$ and $G_{1}$ are given by (3.15) and

$$
\begin{aligned}
& \int_{C_{a b}[0, T]}^{a n f_{q}}(\delta F(\cdot \mid w+x) * \delta G(\cdot \mid w+x))_{q}(y) d \mu(x) \\
& =\int_{C_{a b}[0, T]}^{a n f_{q}} T_{q}^{(p)}\left(\delta F\left(\frac{y+x}{\sqrt{2}} \mid \cdot\right)\right)(w) T_{q}^{(p)}\left(\delta G\left(\frac{y-x}{\sqrt{2}} \mid \cdot\right)\right)(w) d \mu(x) \\
& -\frac{i}{q} \int_{L_{a b}^{2}[0, T]}(u v, b) \exp \left\{\frac{i}{\sqrt{2}}\langle u+v, y\rangle-\frac{i}{4 q}\left((u-v)^{2}, b\right)\right. \\
& \left.+i\left(\frac{i}{2 q}\right)^{1 / 2}(u-v, a)\right\} d f(u) d g(v) .
\end{aligned}
$$


Proof. By using (3.13) and (3.21), we obtain (4.6) above. To establish (4.7), we note that, by the use of (2.15), (3.24), (3.28), and (3.31),

$$
\begin{aligned}
& \int_{C_{a b}[0, T]}^{a n f_{q}}(\delta F(\cdot \mid w+x) * \delta G(\cdot \mid w+x))_{q}(y) d \mu(x) \\
& =-\int_{C_{a b}[0, T]}^{a n f_{q}} \int_{L_{a b}^{2}[0, T]}\langle u, w+x\rangle\langle v, w+x\rangle \\
& \cdot \exp \left\{\frac{i}{\sqrt{2}}\langle u+v, y\rangle-\frac{i}{4 q}\left((u-v)^{2}, b\right)\right. \\
& \left.+i\left(\frac{i}{2 q}\right)^{1 / 2}(u-v, a)\right\} d f(u) d g(v) d \mu(x) \\
& =-\int_{L_{a b}^{2}[0, T]}\left[\left(\langle u, w\rangle+\left(\frac{i}{q}\right)^{1 / 2}(u, a)\right)\left(\langle v, w\rangle+\left(\frac{i}{q}\right)^{1 / 2}(v, a)\right)+\frac{i}{q}(u v, b)\right] \\
& \cdot \exp \left\{\frac{i}{\sqrt{2}}\langle u+v, y\rangle-\frac{i}{4 q}\left((u-v)^{2}, b\right)+i\left(\frac{i}{2 q}\right)^{1 / 2}(u-v, a)\right\} d f(u) d g(v) \\
& =\int_{C_{a b}[0, T]}^{a n f_{q}} \int_{L_{a b}[0, T]} i\left(\langle u, w\rangle+\left(\frac{i}{q}\right)^{1 / 2}(u, a)\right) \exp \left\{i\left\langle u, \frac{y+x}{\sqrt{2}}\right\rangle\right\} d f(u) \\
& \cdot \int_{L_{a b}[0, T]} i\left(\langle v, w\rangle+\left(\frac{i}{q}\right)^{1 / 2}(v, a)\right) \exp \left\{i\left\langle v, \frac{y-x}{\sqrt{2}}\right\rangle\right\} d g(v) d \mu(x) \\
& -\frac{i}{q} \int_{L_{a b}^{2}[0, T]}(u v, b) \exp \left\{\frac{i}{\sqrt{2}}\langle u+v, y\rangle-\frac{i}{4 q}\left((u-v)^{2}, b\right)\right. \\
& \left.+i\left(\frac{i}{2 q}\right)^{1 / 2}(u-v, a)\right\} d f(u) d g(v) \\
& =\int_{C_{a b}[0, T]}^{a n f_{q}} T_{q}^{(p)}\left(\delta F\left(\frac{y+x}{\sqrt{2}} \mid \cdot\right)\right)(w) T_{q}^{(p)}\left(\delta G\left(\frac{y-x}{\sqrt{2}} \mid \cdot\right)\right)(w) d \mu(x) \\
& -\frac{i}{q} \int_{L_{a b}^{2}[0, T]}(u v, b) \exp \left\{\frac{i}{\sqrt{2}}\langle u+v, y\rangle-\frac{i}{4 q}\left((u-v)^{2}, b\right)\right. \\
& \left.+i\left(\frac{i}{2 q}\right)^{1 / 2}(u-v, a)\right\} d f(u) d g(v)
\end{aligned}
$$

for s-a.e. $y \in C_{a b}[0, T]$.

In our next theorem, we obtain the transforms of the convolution product with respect to the second argument of the variations.

THEOREM 4.4. Let $F, G, p, q$, and $w$ be given as in Theorem 4.1. Then for s-a.e. $y \in C_{a b}[0, T]$,

$$
\begin{aligned}
T_{q}^{(p)} & \left((\delta F(y \mid \cdot) * \delta G(y \mid \cdot))_{q}\right)(w) \\
\quad & =T_{q}^{(p)}\left(\delta F\left(y \mid \frac{\cdot}{\sqrt{2}}\right) \delta G\left(y \mid \frac{\cdot}{\sqrt{2}}\right)\right)(w)
\end{aligned}
$$




$$
\begin{aligned}
&- i\left(\frac{i}{q}\right)^{1 / 2} T_{q}^{(p)}\left(\delta F\left(y \mid \frac{\cdot}{\sqrt{2}}\right)\right)(w) \int_{L_{a b}[0, T]}\left(v, \frac{a}{\sqrt{2}}\right) \exp \{i\langle v, y\rangle\} d g(v) \\
&+ i\left(\frac{i}{q}\right)^{1 / 2} T_{q}^{(p)}\left(\delta G\left(y \mid \frac{\cdot}{\sqrt{2}}\right)\right)(w) \int_{L_{a b}[0, T]}\left(u, \frac{a}{\sqrt{2}}\right) \exp \{i\langle u, y\rangle\} d f(u) \\
&+ \frac{i}{2 q} \int_{L_{a b}^{2}[0, T]}[(u, a)(v, a)+(u v, b)] \exp \{i\langle u+v, y\rangle\} d f(u) d g(v), \\
& \int_{C_{a b}[0, T]}^{a n f_{q}}(\delta F(y+x \mid \cdot) * \delta G(y+x \mid \cdot))_{q}(w) d \mu(x) \\
&=T_{q}^{(p)}\left(\delta F\left(\cdot \mid \frac{w}{\sqrt{2}}\right) \delta G\left(\cdot \mid \frac{w}{\sqrt{2}}\right)\right)(y) \\
&+\left(\frac{i}{q}\right)^{1 / 2} \int_{L_{a b}^{2}[0, T]}\left[\left\langle u, \frac{w}{\sqrt{2}}\right\rangle\left(v, \frac{a}{\sqrt{2}}\right)-\left(u, \frac{a}{\sqrt{2}}\right)\left\langle v, \frac{w}{\sqrt{2}}\right\rangle\right. \\
&\left.+\left(\frac{i}{q}\right)^{1 / 2}\left(u, \frac{a}{\sqrt{2}}\right)\left(v, \frac{a}{\sqrt{2}}\right)+\frac{1}{2}\left(\frac{i}{q}\right)^{1 / 2}(u v, b)\right] \\
&+\exp \left\{i\langle u+v, y\rangle-\frac{i}{2 q}\left((u+v)^{2}, b\right)+i\left(\frac{i}{q}\right)^{1 / 2}(u+v, a)\right\} d f(u) d g(v) .
\end{aligned}
$$

Proof. By using (2.11) and (3.30), we obtain (4.9).

Next, we obtain the variation of the convolution product of transforms.

TheOrem 4.5. Let $F, G, p, q$, and $w$ be given as in Theorem 4.1. Then

$$
\begin{aligned}
\delta\left(T_{q}^{(p)}(F) * T_{q}^{(p)}(G)\right)_{-q}(y \mid w) & \\
= & \delta T_{q}^{(p)}\left(F_{2}\left(\frac{\cdot}{\sqrt{2}}\right) G_{2}\left(\frac{\cdot}{\sqrt{2}}\right)\right)(y) \\
=\int_{L_{a b}^{2}[0, T]} & \frac{i}{\sqrt{2}}\langle u+v, w\rangle \\
\cdot & \exp \left\{\frac{i}{\sqrt{2}}\langle u+v, y\rangle-\frac{i}{4 q}\left((u+v)^{2}, b\right)\right. \\
+ & \left.i\left(\frac{-i}{2 q}\right)^{1 / 2}(u-v, a)+i\left(\frac{i}{q}\right)^{1 / 2}(u+v, a)\right\} d f(u) d g(v)
\end{aligned}
$$

for s-a.e. $y \in C_{a b}[0, T]$ where $F_{2}$ and $G_{2}$ are given by (3.19).

Proof. By using (3.17) and the same calculation in the proof of Theorem 3.6, we obtain (4.10).

Now, we obtain the formulas for convolution product of the variation of the transform. There are two cases; namely, we can take the convolution with respect to the first argument or the second argument of the variation. 
THEOREM 4.6. Let $F, G, p, q$, and $w$ be given as in Theorem 4.1. Then, for s-a.e. $y \in C_{a b}[0, T]$,

$$
\left(\delta T_{q}^{(p)}(F)(\cdot \mid w) * \delta T_{q}^{(p)}(G)(\cdot \mid w)\right)_{-q}(y)=T_{q}^{(p)}\left(\delta F_{2}\left(\frac{\cdot}{\sqrt{2}} \mid w\right) \delta G_{2}\left(\frac{\cdot}{\sqrt{2}} \mid w\right)\right)(y),
$$

where $F_{2}$ and $G_{2}$ are given by (3.19); and if $a \in A$, then

$$
\begin{aligned}
\left(\delta T_{q}^{(p)}(F)(y \mid \cdot) * \delta T_{q}^{(p)}(G)(y \mid \cdot)\right)_{-q}(w) \\
=\left(\delta T_{q}^{(p)}(F)\left(y \mid \frac{w}{\sqrt{2}}\right)+\left(-\frac{i}{q}\right)^{1 / 2} \delta T_{q}^{(p)}(F)\left(y \mid \frac{a}{\sqrt{2}}\right)\right) \\
\quad \cdot\left(\delta T_{q}^{(p)}(G)\left(y \mid \frac{w}{\sqrt{2}}\right)-\left(-\frac{i}{q}\right)^{1 / 2} \delta T_{q}^{(p)}(G)\left(y \mid \frac{a}{\sqrt{2}}\right)\right) \\
-\frac{i}{2 q} \int_{L_{a b}^{2}[0, T]}(u v, b) \exp \left\{i\langle u+v, y\rangle-\frac{i}{2 q}\left(u^{2}+v^{2}, b\right)\right. \\
\left.+i\left(\frac{i}{q}\right)^{1 / 2}(u+v, a)\right\} d f(u) d g(v) .
\end{aligned}
$$

Proof. By using (3.22), (3.24), and the same calculation in the proof of Theorem 3.6, we obtain (4.11). Further, proceeding as in the proof of Theorem 3.8 and using (3.33) and (3.22), we have (4.12).

THEOREM 4.7. Let $F, G, p, q$, and $w$ be given as in Theorem 4.1 and let $a \in A$. Then, for s-a.e. $y \in C_{a b}[0, T]$,

$$
\begin{aligned}
\left(T_{q}^{(p)}(\delta F(y \mid \cdot)) * T_{q}^{(p)}(\delta G(y \mid \cdot))\right)_{q}(w) \\
=(\delta F(y \mid \cdot) * \delta G(y \mid \cdot))_{q}(w)-\left(\frac{i}{q}\right)^{1 / 2} \delta F(y \mid a) T_{q}^{(p)}\left(\delta G\left(y \mid \frac{\cdot}{\sqrt{2}}\right)\right)(-w) \\
\quad+\left(\frac{i}{q}\right)^{1 / 2} \delta G(y \mid a) T_{q}^{(p)}\left(\delta F\left(y \mid \frac{\cdot}{\sqrt{2}}\right)\right)(w)+\frac{i}{q} \delta F(y \mid a) \delta G(y \mid a)
\end{aligned}
$$

Proof. By using (3.24) and a direct calculation, we obtain (4.13).

ACKNOWLEDGMENT. The first author was partially supported by Korea Science and Engineering Foundation (KOSEF) in Korea in 2000.

\section{REFERENCES}

[1] M. D. Brue, A functional transform for Feynman integrals similar to the Fourier-transform, Ph.D. thesis, University of Minnesota, 1972.

[2] R. H. Cameron, The first variation of an indefinite Wiener integral, Proc. Amer. Math. Soc. 2 (1951), 914-924.

[3] R. H. Cameron and D. A. Storvick, An $L_{2}$ analytic Fourier-Feynman transform, Michigan Math. J. 23 (1976), no. 1, 1-30.

[4] _ Some Banach algebras of analytic Feynman integrable functionals, Analytic Functions, (Proc. 7th Conf., Kozubnik, Poland, 1979), Lecture Notes in Math., vol. 798, Springer-Verlag, Berlin, 1980, pp. 18-67. 
[5] _ Feynman integral of variations of functionals, Gaussian Random Fields (Nagoya, 1990), Ser. Probab. Statist., vol. 1, World Scientific, New Jersey, 1991, pp. 144-157.

[6] S. J. Chang and D. M. Chung, Conditional function space integrals with applications, Rocky Mountain J. Math. 26 (1996), no. 1, 37-62.

[7] S. J. Chang, S. J. Kang, and D. Skoug, Conditional generalized analytic Feynman integrals and a generalized integral equation, Int. J. Math. Math. Sci. 23 (2000), no. 11, 759776.

[8] D. M. Chung, Scale-invariant measurability in abstract Wiener spaces, Pacific J. Math. 130 (1987), no. 1, 27-40.

[9] D. M. Chung and D. Skoug, Conditional analytic Feynman integrals and a related Schrödinger integral equation, SIAM J. Math. Anal. 20 (1989), no. 4, 950-965.

[10] T. Huffman, C. Park, and D. Skoug, Analytic Fourier-Feynman transforms and convolution, Trans. Amer. Math. Soc. 347 (1995), no. 2, 661-673.

[11] Convolution and Fourier-Feynman transforms, Rocky Mountain J. Math. 27 (1997), no. 3, 827-841.

[12] _ Generalized transforms and convolutions, Int. J. Math. Math. Sci. 20 (1997), no. 1, 19-32.

[13] G. W. Johnson and D. L. Skoug, An $L_{p}$ analytic Fourier-Feynman transform, Michigan Math. J. 26 (1979), no. 1, 103-127.

[14] _ Scale-invariant measurability in Wiener space, Pacific J. Math. 83 (1979), no. 1, 157-176.

[15] _ _ Notes on the Feynman integral. I, Pacific J. Math. 93 (1981), no. 2, 313-324.

[16]_ Notes on the Feynman integral. II, J. Funct. Anal. 41 (1981), no. 3, 277-289.

[17] _ Notes on the Feynman integral. III. The Schroedinger equation, Pacific J. Math. 105 (1983), no. 2, 321-358.

[18] C. Park, D. Skoug, and D. A. Storvick, Relationships among the first variation, the convolution product and the Fourier-Feynman transform, Rocky Mountain J. Math. 28 (1998), no. 4, 1447-1468.

[19] J. Yeh, Stochastic Processes and the Wiener Integral, Pure and Applied Mathematics, vol. 13, Marcel Dekker, New York, 1973.

Seung Jun Chang: Department of Mathematics, Dankook University, Cheonan, 330714, KOREA

E-mail address: sejchang@anseo.dankook.ac.kr

Jae Gil Choi: Department of Mathematics, Dankook University, Cheonan, 330-714, KOREA

E-mail address: jgchoi@anseo.dankook.ac.kr 


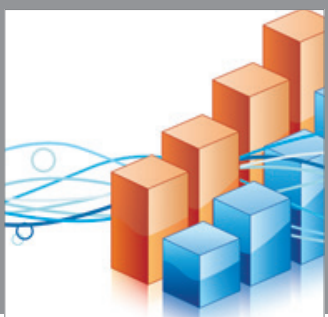

Advances in

Operations Research

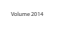

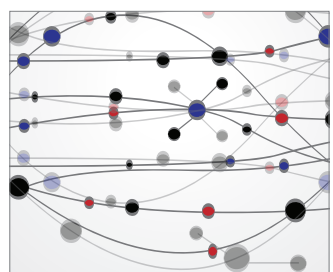

\section{The Scientific} World Journal
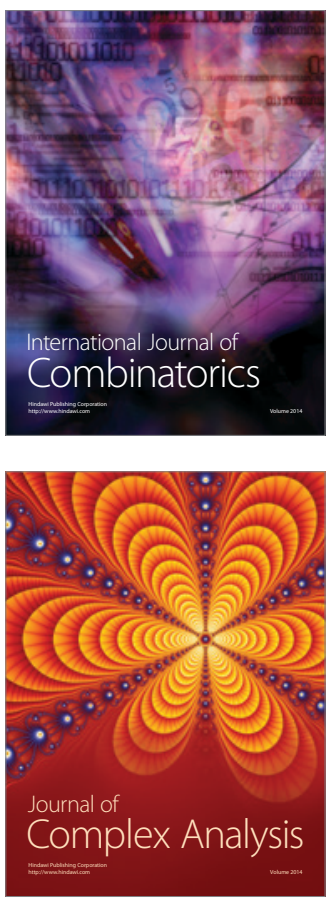

International Journal of

Mathematics and

Mathematical

Sciences
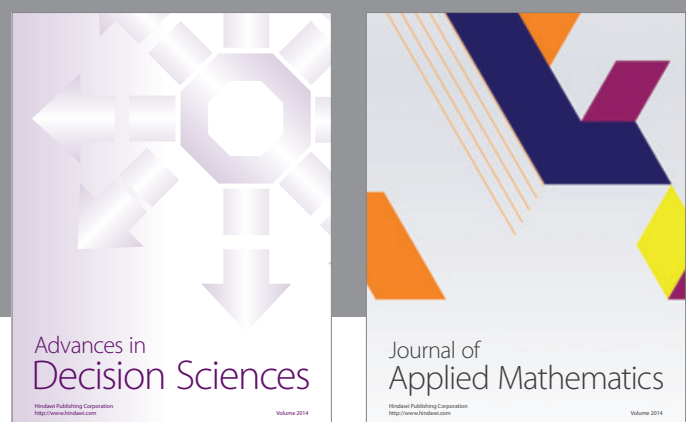

Journal of

Applied Mathematics
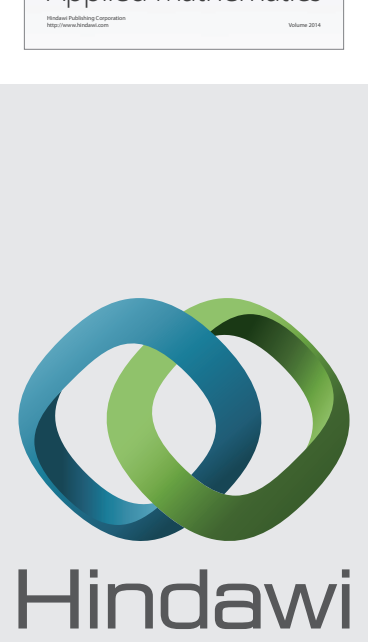

Submit your manuscripts at http://www.hindawi.com
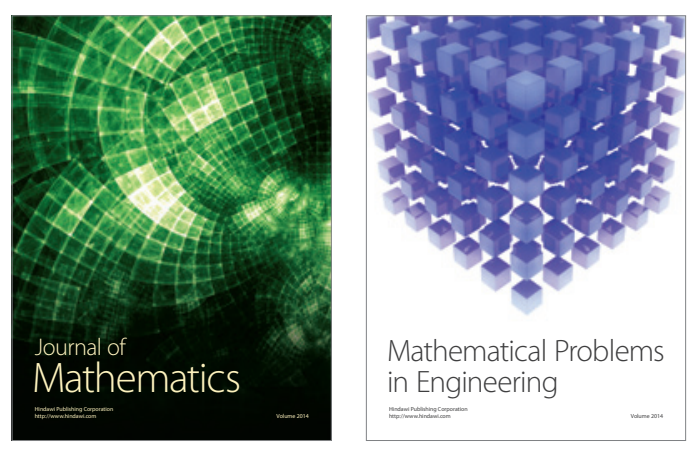

Mathematical Problems in Engineering
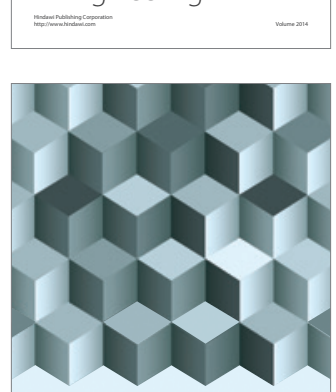

Journal of

Function Spaces
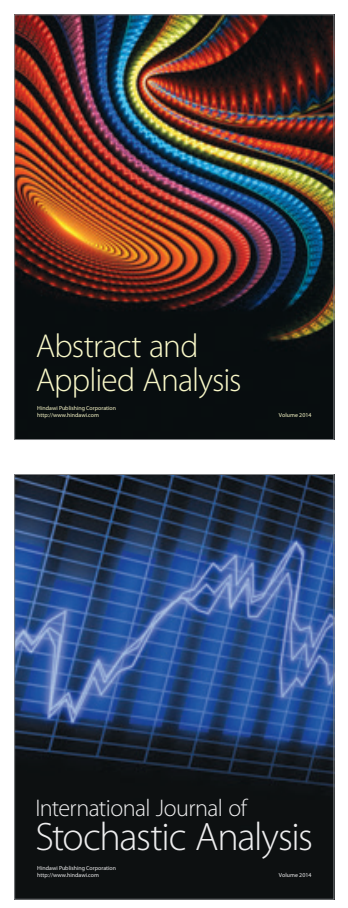

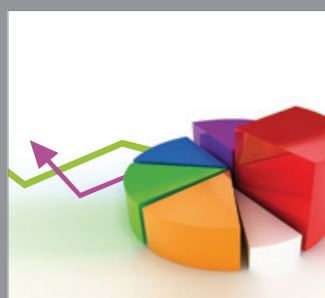

ournal of

Probability and Statistics

Promensencen
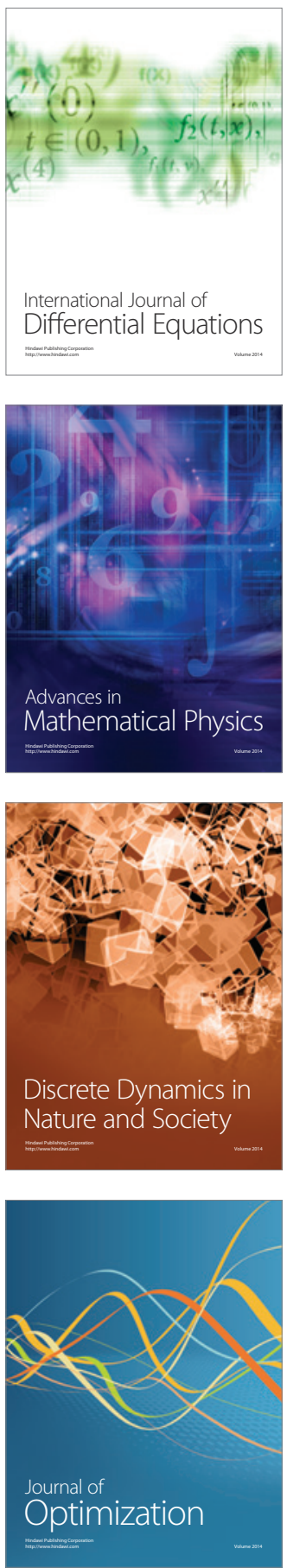